Bio - grafía. Escritos sobre la Biología y su Enseñanza. ISSN 2027-1034

Edición Extraordinaria. p.p. 1088 - 1099

Memorias del IX Encuentro Nacional de Experiencias en Enseñanza de la Biología y la Educación Ambiental. IV Congreso Nacional de Investigación en Enseñanza de la Biología.

\title{
ENSEÑANZA DEL NIVEL TRÓFICO DEL GRUPO CHIRÓPTERA MEDIANTE EL USO DE FICHAS LEGO PARA LA CONSTRUCCIÓN DE BIO-PROTOTIPOS EN LOS GRADOS NOVENOS DEL INSTITUTO PEDAGÓGICO NACIONAL
}

\section{ALEJANDRO GIRALDO MORA ${ }^{1}$}

\section{RESUMEN}

El siguiente artículo de investigación tiene como propósito dar a conocer una ruta educativa en donde el maestro en formación puede construir nuevas ideas para la enseñanza de las ciencias en el aula de clases, la iniciativa y el trabajo de este proyecto nace en la línea de investigación bio-didáctica y recursos educativos. La implementación de este proyecto de investigación pedagógico y didáctico se llevó a cabo en el instituto pedagógico nacional (IPN) con los grados noveno se efectuaron una serie de clases en los meses febrero a mayo del 2017 para enseñar la diversidad del grupo Chiroptera en Colombia y la comprensión de la acción enzimática del dicho orden mediante la construcción de prototipos con fichas lego.

\section{PALABRAS CLAVE:}

Prototipo, enseñanza, Didáctica, murciélagos, nutrición, bloques lego, diversidad.

\section{ABSTRACT}

The following research article aims to present an educational route in which the teacher in training can build new ideas for teaching science in the classroom, initiative and work of this project is born in the line of research Bio-didactics and educational resources. The implementation of this pedagogical and didactic research project was carried out at the National Pedagogical Institute (IPN) with the ninth grade, a series of classes were implemented in the months of February to May of 2017 to teach the diversity of the Chiroptera group in Colombia And the understanding of the enzymatic action of the said order by the construction of prototypes with lego chips.

\section{KEYWORDS:}

Prototype, teaching, Didactics, bats, nutrition, lego blocks, diversity.

\footnotetext{
${ }^{1}$ Estudiante Universidad Pedagógica Nacional.
} 
Bio - grafía. Escritos sobre la Biología y su Enseñanza. ISSN 2027-1034

Edición Extraordinaria. p.p. 1088 - 1099

Memorias del IX Encuentro Nacional de Experiencias en Enseñanza de la Biología y la

Educación Ambiental. IV Congreso Nacional de Investigación en Enseñanza de la Biología.

\section{INTRODUCCIÓN}

El reto que tienen los maestros para la enseñanza de las ciencias naturales en el aula de clase es cada vez más grande, debido a que el contexto de los estudiantes va cambiando conforme avanzan los años y quizá el interés por aprender disminuya drásticamente y es allí donde el maestro debe construir nuevos protocolos de enseñanza que vayan acorde a cada generación, dichos protocolos pueden cambiar los modelos de educación frente a la perspectiva de los estudiantes. Por tal razón la enseñanza de un grupo tan poco estudiado en el aula de clases como los murciélagos mediante la construcción de prototipos a escala con fichas lego pues en el proceso de ensamblar bloques y construir nuevos objetos que sirvan de modelo analógico para la comprensión de un tema específico se torna interesante. De tal forma que los estudiantes conozcan la diversidad de estos mamíferos voladores en el país, siendo los murciélagos "el grupo de mamíferos más diverso y abundante en Colombia, con más de 180 especies registradas y cumplen papeles sumamente relevantes en el ambiente". (Castaño y Botero, 2004).

\section{OBJETIVOS}

\section{General:}

-Aportar a la construcción del conocimiento biológico de los estudiantes mediante la construcción de un prototipo tangible hecho con bloques lego.

\section{Específicos:}

-Dar a conocer a los estudiantes del IPN una nueva estrategia de aprendizaje en el área ciencias mediante la utilización de bloques lego.

-Establecer momentos de debate y reflexión a medida que los estudiantes construyen prototipos para la comprensión de la diversidad.

-Fortalecer los conocimientos que se adquieren en el proceso de construcción del prototipo hecho con fichas lego.

\section{PROBLEMÁTICA:}

Desde segundo semestre del año 2016 en el mes de agosto, se hizo un acercamiento al instituto pedagógico nacional, (IPN) escenario educativo y por lo tanto el espacio donde los estudiantes interactúan con los maestros en formación, los maestros en ejercicio y sus compañeros, en el proceso de contextualización se 


\section{Bio - grafía. Escritos sobre la Biología y su Enseñanza. ISSN 2027-1034}

\section{Edición Extraordinaria. p.p. 1088 - 1099}

Memorias del IX Encuentro Nacional de Experiencias en Enseñanza de la Biología y la Educación Ambiental. IV Congreso Nacional de Investigación en Enseñanza de la Biología.

evidenció la capacidad de aprendizaje por parte de los estudiantes. Este proyecto está enfocado hacia la enseñanza de la diversidad nutricional a través del uso del modelo mental y la construcción de prototipo tangibles, siendo esta una extensión del conocimiento, un apoyo tangible para la compresión de las ciencias biológicas en el aula de clase.

En el campo educativo se evidencian fallas por parte de los maestros a la hora de enseñar contenidos propios de sus áreas, el maestro debe impulsar a su estudiante, darle un "empujón" con buenas bases previas y de esta manera pueda establecer relaciones con lo que ya ha aprendido y lo que está aprendiendo, Es por esto que cada estudiante puede tener un acercamiento al campo biológico mediante la elaboración de prototipos, en este caso el uso de fichas lego, un objeto lúdico y no didáctico $Y$ que en este proyecto se implementó como herramienta de enseñanza y no como juego tradicional irlandés, puesto que de ahí el origen de su palabra, etimologicamente es la abreviatura de dos palabras danesas: "leg godt" (cuyo significado es "jugar bien").

Se planteó entonces la siguiente pregunta problema: ¿Cómo la construcción mediante el uso de bloques lego de un prototipo biológico favorece la construcción del conocimiento biológico en el aula de clases?

La implementación de bloques lego para la enseñanza, es una estrategia innovadora que se ha implementado para comprender áreas como las matemáticas enseñando a estudiantes de todas las edades. Lego, siendo una gran corporación de orden internacional presenta un enfoque educativo. "lego education" donde se pretende estudiar matemáticas, tecnología, ingeniería, ciencia y arquitectura, la enseñanza de soluciones para la escuela media es base fundamental para crecer en habilidades de pensamiento crítico e imaginación. "la solución creativa de los estudiantes de secundaria, las habilidades de resolución de problemas y que puedan convertirse en los pensadores críticos y creadores del futuro. Lego plantea soluciones para la educación apoyar sus esfuerzos de enseñanza con estrategias de enseñanza estructuradas y el plan de estudios correspondiente, enfocadas para la enseñanza de la ciencia, tecnología, ingeniería y matemáticas. Estas soluciones permiten a los estudiantes entender temas desafiantes, animarles a desarrollar habilidades de pensamiento crítico, desarrollar sus ideas y materializar sus propias ideas a través de experiencias de aprendizaje didáctico" (Lego, Group. (2017). Por otro lado se presenta una propuesta diferente donde se pretende que los estudiantes construyan habilidades útiles para el siglo $X X I$, explorando los temas de la vida real y los desafíos que esta presenta, aportando al crecimiento de un pensamiento crítico e imaginativo del estudiante y en cuanto a los maestros simplificar la planificación de sus clases, haciéndolas más didácticas y de esta manera captar la atención de sus estudiantes. En el proyecto de investigación se planteó la construcción de un prototipo para la 
Bio - grafía. Escritos sobre la Biología y su Enseñanza. ISSN 2027-1034

Edición Extraordinaria. p.p. 1088 - 1099

Memorias del IX Encuentro Nacional de Experiencias en Enseñanza de la Biología y la

Educación Ambiental. IV Congreso Nacional de Investigación en Enseñanza de la Biología.

comprensión de la acción enzimática expuesta en los modelos de Koshland y Fisher que expresa la afinidad de las enzimas y los sustratos al interior del organismo, por un lado Daniel Koshland habla de un modelo de ajuste inducido para la interacción enzima-sustrato en donde la enzima cambia de forma de la unión con el sustrato, mientras que el modelo de Emil Fisher, el centro activo de la enzima es complementario a la forma del sustrato y por eso adopta el nombre de modelo llave y cerradura. Para la enseñanza de la biología, en este caso puede revelar la gran diversidad nutricional de los murciélagos frente a los recursos disponibles en el ambiente, esto con el fin de desmitificar la concepción frente a esta clase de organismos.

\section{JUSTIFICACIÓN}

El uso del modelo mental y la construcción de prototipos tangibles con bloques lego para la enseñanza de la biología puede ser un elemento educativo, el ser humano aprende cuando modela en su mente, es como si construyera un esquema para comprender la realidad y una vez este esquema, modelo o ruta mental esta aprendido, se dice que el sujeto ha interiorizado el concepto que se enseña. "El modelo mental se construye mediante la modelización, siendo el modelo una interiorización abstraída de la realidad, que puede ser abstracta y que por medio del modelo mental esta suele comprenderse mejor haciendo una trasposición o re contextualización didáctica" (Chevallard, 1985). Para comprender el proceso de construcción de un modelo mental es necesario hacer aclaraciones frente a la diferencia etimológica del concepto de modelo mental y la modelización, entendiendo que el modelo mental es el producto de una modelización exhaustiva en donde se construyen varios elementos que facilitan la comprensión de un tema específico, mientras que la modelización es el proceso para llegar al producto de modelo. Se entiende por modelo mental analógico de un sujeto cuando cognitivamente construye una serie de esquemas para comprender un evento en su entorno, en ciencia dice Moreira, 2002, es frecuente utilizar el modelo metal para que los estudiantes comprendan procesos físicos o fenómenos naturales, "Los modelos mentales son análogos estructurales del mundo; su estructura, y no su aspecto, corresponde a la estructura de la situación que representan." (Moreira y Greca, 1998). El modelo mental puede utilizarse para razonar, en pocas palabras el proceso de resolución de problemas se da gracias a la construcción de varias ideas y que al converger dan a la luz una idea o perspectiva clara que hace al sujeto comprender lo que sucede a su alrededor y por ende dar una respuesta a un problema cognitivo extraído de la realidad por parte del estudiante. 


\section{Bio - grafía. Escritos sobre la Biología y su Enseñanza. ISSN 2027-1034}

\section{Edición Extraordinaria. p.p. 1088 - 1099}

Memorias del IX Encuentro Nacional de Experiencias en Enseñanza de la Biología y la Educación Ambiental. IV Congreso Nacional de Investigación en Enseñanza de la Biología.

Las posibilidades de desarrollar un modelo mental en el aula de clases se hace eficiente debido a que el proceso de aprendizaje varía dependiendo el usuario, Moreira afirma que "Los modelos mentales son finitos en tamaño y no pueden representar directamente un dominio infinito. No obstante, un único modelo mental puede representar un número infinito de posibles estados de cosas pues ese modelo puede ser revisado recursivamente". (Moreira, 2002. Pag.7). Los modelos mentales son funcionales y prácticos, por ejemplo para la comprensión de un tema como la nutrición en seres vivos, se habla al grupo de estudiantes que hay un patrón de regulación el cual tiene unas serie de procesos al interior del organismo y que este es vital para el desarrollo del mismo, para el progreso de la clase, el sujeto que aprende hace representaciones cognitivas para poder comprender a cabalidad, esto dice Moreira citando a Norman, 1983 dice que los modelos mentales de un individuo son limitados por factores tales como su conocimiento y su experiencia previa con sistemas semejantes, así como por la propia estructura del sistema de procesamiento de información del ser humano, es por esto, que desde este proyecto de investigación se plantea la utilización del modelo mental para la construcción de un prototipo tangible en la enseñanza de conceptos biológicos, retomando al ejemplo de la clase de nutrición, el profesor tiene a su favor que el estudiante comprenda el concepto de proceso, siendo este una secuencia de pasos dispuesto con algún tipo de lógica, entonces, este plantearía la elaboración de un prototipo como una "Maquina de Goldberg" para explicar por medio de ésta el proceso de nutrición y al mismo tiempo en la interacción enseñanza y aprendizaje se construya un modelo mental para comprender el patrón de nutrición.

"El reto para el maestro es el de integrar los contenidos, propósitos, estrategias, prototipos didácticos y prácticas que favorecen a los objetivos generales y específicos de la materia de Física. Y, así el alumno sea el beneficiado. Ante estas intenciones nos planteamos realizar esta innovación en la materia de Física como un reto personal para obtener una calidad en la educación." (Hernández, 2005).

Los prototipos entonces para este proyecto, brindarían un aporte a los estudiantes que aprenden biología en las instituciones educativas para comprender, entender, interiorizar y relacionar los principios biológicos vistos en el aula de clase con un objeto didáctico y con la vida cotidiana. Los prototipos en el aula de clase permitirán que la relación del estudiante con los contenidos biológicos tenga más sentido para él, esta estrategia innovadora presenta una relación entre los conceptos previos y los nuevos, de esta manera puede ir aprendiendo cada contenido con mayor facilidad desmitificando la postura de muchos al mencionar que un prototipo solo es un juego, Hernández, 2005 menciona que uno de los postulados del constructivismo dice que los estudiantes que aprende cuando es 
Bio - grafía. Escritos sobre la Biología y su Enseñanza. ISSN 2027-1034

Edición Extraordinaria. p.p. 1088 - 1099

Memorias del IX Encuentro Nacional de Experiencias en Enseñanza de la Biología y la

Educación Ambiental. IV Congreso Nacional de Investigación en Enseñanza de la Biología.

capaz de construir un conocimiento acerca de un objeto de la realidad o algún contenido por aprender.

\section{APORTES E INSTRUMENTOS}

La construcción de estos prototipos con la ayuda de bloques lego, puede convertirse en una nueva ruta de enseñanza debido a que los estudiantes pueden crear diferentes modelos alternativos para comprender un tema específico en el aula de clase, en pocas palabras los estudiantes aprenden construyendo.

Este trabajo se realizó desde el paradigma hermenéutico interpretativo con un método cualitativo. Los instrumentos de investigación son entrevistas, encuestas y un taller final antes de la implementación para comprender a fondo las expectativas que presentan los estudiantes frente al proyecto, estas contienen preguntas orientadoras que ayudan a la comprensión del tema. Las categorías de sistematización y análisis de los datos obtenidos en las entrevistas son: Lenguaje, conocimiento biológico, modelos mentales, prototipos y modelización. Bajo los códigos: EN: encuestas, TL: taller ET: entrevistas acompañado por el número de encuesta. EA: estudiante y apellido y PG: pregunta y se asocia al numeral correspondiente en la lista de la entrevista.

El taller estuvo enfocado hacia la comprensión de la acción enzimática y los procesos de nutrición en el orden Chiroptera y cómo se evidencia la diversidad frente al recurso disponible en un ambiente determinado. También se hizo un histograma que recopiló los datos desde el inicio del proyecto y su culminación analizando y comparando resultados. Según avanzó el semestre con base en la información recopilada en las encuestas, entrevistas y el taller se evidenciaron fortalezas y debilidades de esta manera se trianguló dicha información. A la hora de implementar un proyecto bajo este enfoque.

Se realizó la entrega de una encuesta previa a la implementación del taller con lego, con el fin de brindar información al maestro en formación frente al tema que se expuso en el taller, todo el contenido estuvo relacionado frente a la comprensión de la acción enzimática y así hacer un sondeo frente a las concepciones y conocimientos frente al tema, posteriormente se entregó la encuesta, para conocer ciertas concepciones de los estudiantes, una vez el taller se terminó se hizo una comparación de los resultados para comprobar si en realidad la construcción del bio-prototipo es factible para la enseñanza de la biología, en este caso para la enseñanza de la nivel trófico del grupo Chiroptera.

Se realizó un censo antes de implementar el taller propuesto para la construcción del conocimiento biológico a través de la construcción del bio-prototipo, esto con el 
Bio - grafía. Escritos sobre la Biología y su Enseñanza. ISSN 2027-1034

Edición Extraordinaria. p.p. 1088 - 1099

Memorias del IX Encuentro Nacional de Experiencias en Enseñanza de la Biología y la

Educación Ambiental. IV Congreso Nacional de Investigación en Enseñanza de la Biología.

fin de asegurar la comprensión del tema "acción enzimática" puesto que muchos de los estudiantes solo presentan la concepción de que los murciélagos son hematófagos solamente, pero en realidad al realizar un estudio más minucioso donde se aclaran dudas acerca de su diversidad nutricional. Se realizó una encuesta la cual contenía preguntas hacia la población estudiantil (curso 901 con un total de 25 estudiantes) , para comprender su hábito nutricional y el conocimiento acerca de las enzimas y en donde se podrían evidenciar. La encuesta solo presentaba tres preguntas básicas y se recolectó la siguiente información:

\begin{tabular}{|c|c|c|}
\hline ENCUESTA 1 & OPCIONES & $\mathbf{N}^{0}$ \\
\hline \multirow{3}{*}{ Pregunta 1} & Carnes & $\overline{14}$ \\
\hline & Frutas & 3 \\
\hline & Lácteos & 8 \\
\hline \multirow{3}{*}{ Pregunta 2} & Átomo & 9 \\
\hline & Enzima & 4 \\
\hline & Virus & 12 \\
\hline \multirow{4}{*}{ Pregunta 3} & Lácteos & 8 \\
\hline & Dulces & 0 \\
\hline & Frutas & 4 \\
\hline & Ninguna & 13 \\
\hline
\end{tabular}

Fig 1. Al tabular los resultados del censo los datos arrojaron cierta información relevante para la investigación en el aula de clase, para la pregunta 1 la mayoría de estudiantes equivalentes a un 56\% dice que las enzimas se encuentran en su totalidad en carnes, también se evidencia la falta de conocimiento frente a la 
Bio - grafía. Escritos sobre la Biología y su Enseñanza. ISSN 2027-1034

Edición Extraordinaria. p.p. 1088 - 1099

Memorias del IX Encuentro Nacional de Experiencias en Enseñanza de la Biología y la

Educación Ambiental. IV Congreso Nacional de Investigación en Enseñanza de la Biología.

estructura de una enzima, puesto que los estudiantes asociaban más la estructura de una enzima con la de un átomo o un virus. se encuentra un paralelo entre la afectación de la población frente a ciertos nutrientes, causando malestares, donde un $32 \%$ de la población padece de intolerancia a la lactosa y un $16 \%$ a frutas, para un total de $48 \%$.en contraste con un $52 \%$ de la población que no presentaba ninguna sensibilidad frente algún nutriente.

Se analiza con el cuadro (fig1) al obtener los resultados, se hizo más viable enfocar la ejecución del taller proyectado hacia la enseñanza de la acción enzimática, con el fin de aclarar dudas y construir cocimiento frente a la concepción de los murciélagos y sus hábitos nutricionales y como este conocimiento aportaría a la comprensión del por qué ciertos organismos padecen ciertas intolerancias a nutrientes en el medio.

\section{ANÁLISIS DE RESULTADOS}

La información que se analiza a continuación es producto de la implementación de los instrumentos de investigación, ya mencionados como encuestas, entrevistas y el taller en donde se construyó el bio-prototipo que simula la interacción enzimasustrato. Luego de hacer el respectivo conteo de respuestas de las encuestas se generaron gráficos que contienen la información plasmada en porcentajes, y a continuación se presenta la interpretación de los datos obtenidos. En la encuesta se establece una división de las opciones si y no para mayor orden y lectura de datos. La encuesta 2 se realizó virtualmente donde los estudiantes respondían desde su ordenador en la sala de informática de la institución.

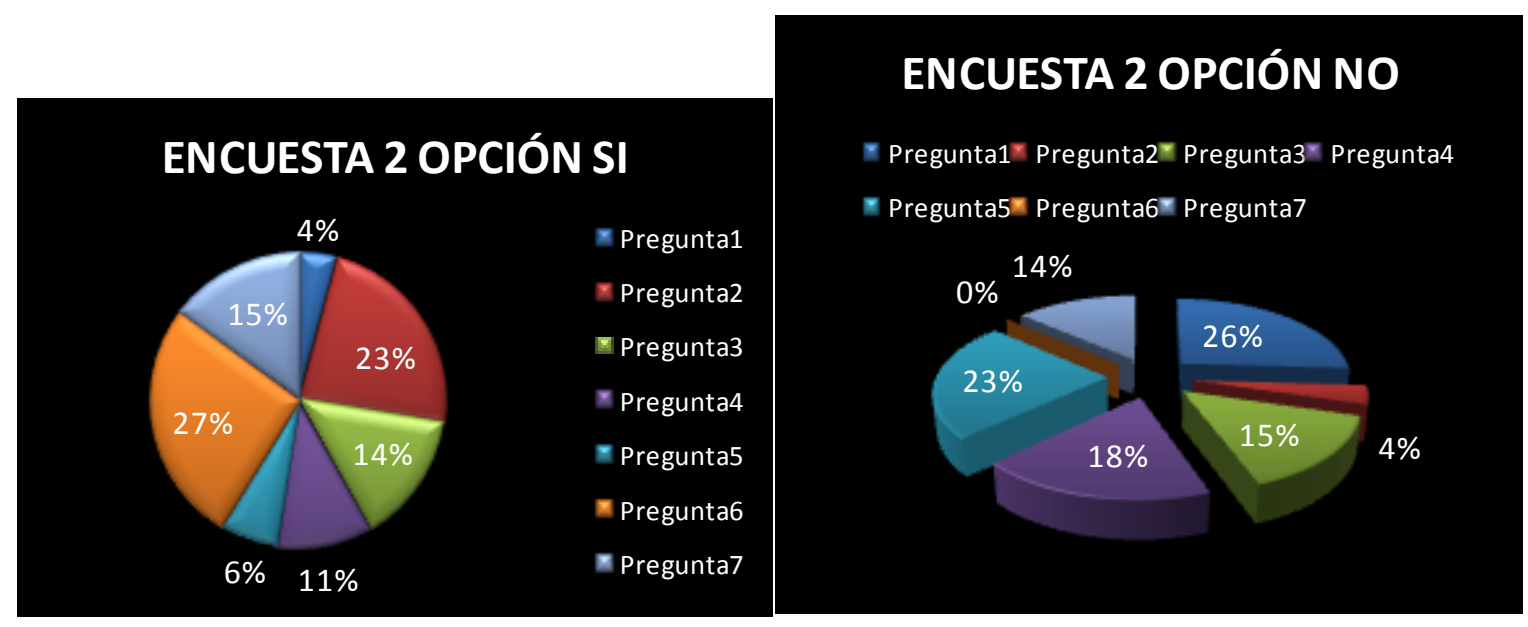

Fig. 2. Se presentan los porcentajes producto de la encuesta 2. La tabulación de datos se ha dividido para una mayor lectura e interpretación de los resultados. 
Bio - grafía. Escritos sobre la Biología y su Enseñanza. ISSN 2027-1034

Edición Extraordinaria. p.p. 1088 - 1099

Memorias del IX Encuentro Nacional de Experiencias en Enseñanza de la Biología y la

Educación Ambiental. IV Congreso Nacional de Investigación en Enseñanza de la Biología.

En esta segunda encuesta, reveló al maestro, la información adecuada para construir el taller investigativo enfocado hacia la comprensión de la acción enzimática y como está demuestra la diversidad nutricional en el grupo Chiroptera. En la opción si del cuestionario, se evidenció en las preguntas: 1, 4 y 5 (1. ¿Sabes como funciona el modelo enzimático de Fisher y Koshland?/ 4. ¿Sabes por que se producen ciertas intolerancias frente a nutrientes contenidos en los alimentos? / 5. ¿Sabes que es una enzima y para que sirve? ) un bajo conocimiento frente al modelo enzimático planteado por koshland y Fisher que explica la acción enzimática y la interacción de ciertos nutrientes al interior del organismo, el por qué se producen ciertas intolerancias frente a nutrientes que se consumen a diario y cuál sería la relación taxonómica por pertenecer a la clase Mammalia tanto humanos como murciélagos. Por otro lado también se evidencia que los estudiantes presentan cierta curiosidad por aprender un tema nuevo que explique, como se evidencia en la pregunta número 6 donde se le pregunta: ¿Te interesaría conocer más acerca de por qué se producen ciertas intolerancias? Con u resultado del $27 \%$ en sondeo general, acompañado de la pregunta número 2 la cual le pregunta al estudiante: ¿te interesaría conocer el modelo de acción enzimática de Fisher y koshland? Dando una respuesta del $23 \%$ de estudiantes con interés de aprender el tema de acción enzimática vs un $4 \%$ al cual el tema quizá es de suma relevancia.

Al terminar la implementación del taller para la construcción del bio-prototipo para la comprensión dela acción enzimática y así aprender acerca de la diversidad nutricional de los murciélagos, se hizo entrega de una encuesta para evaluar el proceso del taller y el desarrollo de los estudiantes frente a la construcción del modelo, en paralelo con la segunda encuesta donde se evidenciaba un vacío conceptual frente al conocimiento biológico, esta vez los datos obtenidos resaltan la aceptación de los estudiantes frente al desarrollo y el contenido del taller, las opciones elegidas por los estudiantes en las cuatro primeras preguntas demuestran un desempeño superior y alto, relacionadas con la utilidad del curso para el desarrollo y construcción del conocimiento biológico, evaluación de los contenidos y el tiempo en el que se realizó la implementación del proyecto. 
Bio - grafía. Escritos sobre la Biología y su Enseñanza. ISSN 2027-1034

Edición Extraordinaria. p.p. 1088 - 1099

Memorias del IX Encuentro Nacional de Experiencias en Enseñanza de la Biología y la Educación Ambiental. IV Congreso Nacional de Investigación en Enseñanza de la Biología.

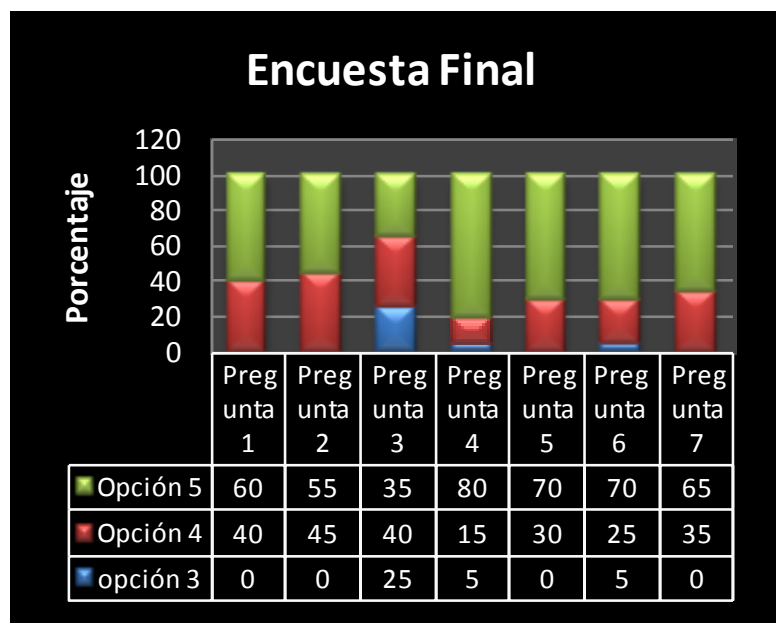

Fig.3 Se recomienda observar los anexos para contrastar la información con las preguntas y las opciones a evaluar por los estudiantes. Donde: La opción 1: bajo, 2: básico, 3: Alto 4: Superior 5: Excelente.

Las preguntas 5,6 y 7 relacionadas con el desarrollo de la clase, orden, materiales y metodología, claridad en el tema, los estudiantes demuestró una aceptación evidente, respaldada con casi un $80 \%$ en la calificación de la opción 5(Excelente) Por lo tanto el desarrollo y construcción del prototipo para la enseñanza de la biología es un elemento innovador como recurso educativo en el aula de clases. Se hizo entrega de una entrevista con preguntas abiertas para conocer la opinión del grupo., para el análisis de los datos se realizó una triangulación de instrumentos para el análisis de datos cualitativos, esto se utiliza para ver la realidad del contexto educativo desde diferentes puntos de vista, el siguiente cuadro analítico, para dicha triangulación verifica la información recolectada,(Ver Anexos) desde la triangulación se relacionan tanto la pregunta problema como el objetivo general del proyecto, se identifican los elementos clave recolectados en los tres instrumentos de investigación (encuestas, entrevista, taller).

\section{CONCLUSIONES}

-Como resultado del proyecto de investigación educativa se concluye que los vacíos epistemológicos de los estudiantes frente a los conceptos biológicos son evidentes, no obstante la enseñanza de la biología utilizando un recurso educativo 
Bio - grafía. Escritos sobre la Biología y su Enseñanza. ISSN 2027-1034

Edición Extraordinaria. p.p. 1088 - 1099

Memorias del IX Encuentro Nacional de Experiencias en Enseñanza de la Biología y la

Educación Ambiental. IV Congreso Nacional de Investigación en Enseñanza de la Biología.

sirve de extensión cognitiva y así el estudiante pueda comprender mejor en el aula de clase.

-El leguaje de los profesores en ejercicio y los estudiantes son diferentes, esto produce en el escenario educativo una ruptura entre la comunicación de los sujetos y por lo tanto imposibilita el proceso de enseñanza y aprendizaje, es por esto que el uso de modelos mentales, el proceso de modelización y la materialización de las ideas para construir un prototipo hace que converja el lenguaje y se construya conocimiento.

- Los prototipos, brindarían un aporte a los estudiantes que aprenden biología en las instituciones educativas para comprender, entender, interiorizar y relacionar los principios biológicos vistos en el aula de clase con un objeto didáctico y con la vida cotidiana.

-El uso de material innovador en el aula de clase rompe el paradigma tradicionalista que imposibilita el desarrollo de la creatividad y la imaginación de los estudiantes, bases fundamentales para el desarrollo de su proceso de aprendizaje.

\section{BIBLIOGRAFÍA:}

-BERG. J. STRYLER. L. TYMOCZKO. J. (2007) BIOQUÍMICA.

-CASTAÑO. J. BOTERO.J. (2004) MURCIELAGOS EN LA ZONA CAFETERA COLOMBIANA

-CHEVALLARD.Y. (1985) LA TRANSPOSICIÓN DIDÁCTICA DEL SABER SABIO AL SABER ENSEÑADO. 
Bio - grafía. Escritos sobre la Biología y su Enseñanza. ISSN 2027-1034

Edición Extraordinaria. p.p. 1088 - 1099

Memorias del IX Encuentro Nacional de Experiencias en Enseñanza de la Biología y la

Educación Ambiental. IV Congreso Nacional de Investigación en Enseñanza de la Biología.

-GRECA.IMOREIRA.M. (1998). MODELOS MENTALES, MODELOS CONCEPTUALES YMODELIZACIÓN.

-HERNANDEZ.V.(2005) LOS PROTOTIPOS DIDÁCTICOS: OTRA FORMA DE ENSEÑAR LA ASIGNATURA DE FÍSICA EN L.A ESCUELA PREPARATORIA DE SILAO DE LA UNIVERSIDAD DE GUANAJUATO.

-LEGO, G. (2017) LEGO EDUCATION. MIDDLE SCHOOL. RECUPERADO DE HTTPS://EDUCATION.LEGO.COM/EN-US/MIDDLE-SCHOOL/EXPLORE.

-MORE IRA. M (2002). MODELOS MENTALES Y MODELOS CONCEPTUALES EN LA ENSEÑANZA \& APRENDIZAJE DE LAS CIENCIAS. TEJINA, LA LAGUNA, STA. CRUZ DE TENERIFE, ESPAÑA.

-NORMAN, D.A. (1983). SOME OBSERVATIONS ON MENTAL MODELS. IN GENTNER, D. AND STEVENS, A.L. (EDS.). MENTAL MODELS. HILLSDALE, NJ: LAWRENCE ERLBAUM ASSOCIATES. 